\title{
Computational Simulation of a Heavy Vehicle Trailer Wake
}

\author{
J. M. Ortega, T. Dunn, R. McCallen, K. Salari
}

This article was submitted to the United Engineering Foundation, Aerodynamics of Heavy Vehicles: Trucks, Buses, and Trains Conference, Monterey, CA, December 2-6, 2002

\section{December 4, 2002}

Lawrence

Livermore

National

Laboratory 
This document was prepared as an account of work sponsored by an agency of the United States Government. Neither the United States Government nor the University of California nor any of their employees, makes any warranty, express or implied, or assumes any legal liability or responsibility for the accuracy, completeness, or usefulness of any information, apparatus, product, or process disclosed, or represents that its use would not infringe privately owned rights. Reference herein to any specific commercial product, process, or service by trade name, trademark, manufacturer, or otherwise, does not necessarily constitute or imply its endorsement, recommendation, or favoring by the United States Government or the University of California. The views and opinions of authors expressed herein do not necessarily state or reflect those of the United States Government or the University of California, and shall not be used for advertising or product endorsement purposes.

This work was performed under the auspices of the U.S. Department of Energy by University of California, Lawrence Livermore National Laboratory under Contract W-7405-Eng-48. 


\title{
Computational Simulation of a Heavy Vehicle Trailer Wake
}

\author{
Jason M. Ortega, Tim Dunn, Rose McCallen, and Kambiz Salari \\ Lawrence Livermore National Laboratory: ortega17@llnl.gov
}

To better understand the flow mechanisms that contribute to the aerodynamic drag of heavy vehicles, unsteady large-eddy simulations are performed to model the wake of a truncated trailer geometry above a no-slip surface. The truncation of the heavy vehicle trailer is done to reduce the computational time needed to perform the simulations. Both unsteady and time-averaged results are presented from these simulations for two grids. A comparison of velocity fields with those obtained from a wind tunnel study demonstrate that there is a distinct difference in the separated wake of the experimental and computational results, perhaps indicating the influence of the geometry simplification, turbulence model, boundary conditions, or other aspects of the chosen numerical approach.

\section{Introduction}

Over the past several decades, a significant amount of effort has been put forth to reduce the aerodynamic drag of heavy vehicles. It has been shown that the power required to overcome the aerodynamic drag at highway speeds (70 $\mathrm{mph}$ ) is on the order of $65 \%$ of the total fuel consumed by the engine of a heavy vehicle. Thus, a reduction in the total aerodynamic drag will have a significant improvement on the fuel economy of a heavy vehicle. The aerodynamic drag can be divided into two components: viscous drag and pressure drag. For the flow over a heavy vehicle at highway speeds, the Reynolds number is large enough such that viscous forces can be safely ignored. Consequently, the drag experienced by a heavy vehicle is primarily due to pressure drag. The pressure drag is comprised of the pressure forces that exist on the front and rear of the vehicle. In recent years, the pressure drag on the front of a heavy vehicle has been successfully reduced by streamlining the tractor, installing air shields on the tractor roof, or optimizing the relative height of the tractor and trailer. However, the pressure drag on the rear of a heavy vehicle, also known as base-drag, yet remains to be reduced on a widespread basis. This 
is due, in part, to the function of a heavy vehicle, which is designed to carry as much cargo as possible. Thus, the shape of the cargo-carrying portion of the heavy vehicle is boxy in nature with many sharp edges. While this shape allows for a large cargo volume and easy access into the cargo bay, it poses a major problem to streamlining the base of the heavy vehicle. Numerous concepts have been proposed in the effort to reduce the base-drag of heavy vehicles. Some of these concepts are passive in nature and include such designs as boattail plates [1], ogives [2, 3], vortex generators [4], and turning vanes [5]. Other concepts are active in nature and include such designs as blowing devices [6]. Despite the large number of proposed solutions, none of them have been implemented on a widespread basis on today's commercial, heavy vehicle fleets. The reason for this is that although some of these concepts can reduce the base-drag by up to $10 \%$, their design renders these concepts impractical, unreliable, or too costly to maintain for use on a long-term basis. It is therefore evident that the successful reduction of heavy vehicle base-drag will require a better understanding of not only the physical mechanisms that contribute to base-drag, but also the needs of heavy vehicle manufacturers and customers. Only then, will it be possible to design and implement an effective base-drag reduction concept.

As a first step in achieving this goal, a computational study is performed on the base of a heavy vehicle geometry. Due to limitations in the number of elements that could be used for timely completion of the simulations, a simplified heavy vehicle geometry and flow domain are utilized. The purpose of this paper is to present the results of this study and to make comparisons with experimental data that were obtained from wind tunnel measurements. The paper is laid out as follows. The vehicle geometry and computational setup are presented in Section 2. Analysis of the unsteady and time-averaged three-dimensional flow fields is given in Section 3. A comparison of the results from these simulations and those from experimental data is made in Section 4. The conclusions and suggestions for future work follow in Section 5.

\section{Computational Setup}

An experimental test [7] investigated the aerodynamics of a $1 / 8$ th scale Ground Transportation System (GTS) (Figure 1) in the NASA Ames $7 \mathrm{ft}$ $\times 10 \mathrm{ft}$ wind tunnel. The purpose of this test was to provide experimental data for the body forces, surface pressures, surface shear stresses, and threedimensional velocity fields in the wake of the GTS for use in validating computational fluid dynamics (CFD) simulations. In the present computational study, we perform fluid dynamic simulations on a simplified GTS geometry and make comparisons with the experimental data.

The GTS geometry and flow domain are simplified in the following manner. As shown in Figure 1b, the GTS geometry is truncated one height dimension upstream of the model base. Additionally, the wind tunnel confinement of the 
GTS model is neglected and the simulations are performed with the truncated GTS positioned above a two-dimensional ground plane. Through these simplifications, the number of elements in the computational domain can be significantly reduced, resulting in a reduction of the computational resources required to perform this investigation. The resulting computational domain is shown in Figure 2. One obvious drawback of this problem simplification is that the resulting geometry may be too far removed from that studied in the wind tunnel tests. Therefore, future investigations must investigate the influence of model truncation and wind tunnel confinement on the results presented in this study.

The unsteady, three-dimensional velocity and pressure fields are calculated with a Galerkin finite-element scheme [8]. The computed pressure and velocity fields are first- and second-order accurate in space, respectively. The simulations are performed using grids that have brick elements. Two fully, unstructured grids are employed in the flow simulations: a coarse grid $(384,000$ elements) and a fine grid (1,054,000 elements). The minimum wall normal spacing of both grids gives $y^{+}$values on the surface of the truck that are of order 10. In refining the coarse grid to the fine grid, additional elements are placed around the periphery of the truncated GTS geometry to better capture the shear layers being shed by the GTS. Figure 3 shows a comparison of the coarse and fine grids.

The surfaces of the ground plane and truncated GTS are treated with a noslip velocity boundary condition. At the inlet to the computational domain, turbulent velocity profiles are specified on the ground plane and the GTS, where the turbulent velocity profiles are given by

$$
\frac{u}{U_{o}}=\left(\frac{y}{\delta}\right)^{1 / 7}
$$

where $U_{o}=92.65 \mathrm{~m} / \mathrm{s}$ is the freestream velocity. $\delta$ is taken to be the turbulent boundary layer thickness on a flat plate with a length of $l=6.26 \mathrm{w}$ (see Figure $1 b)$ and is found from

$$
\delta=0.37 l\left(U_{o} l / \nu\right)^{-1 / 5}
$$

where $w$ is the width of the GTS model. Outside of the boundary layer, the velocity specified at the inlet is $U_{o}$, yielding a width-based Reynolds number, $U_{o} w / \nu$, of $2.0 \times 10^{6}$, where $\nu$ is the kinematic viscosity of air. On the top, sides, and outlet faces of the computational domain, a zero natural boundary condition is specified [8]. A large eddy simulation (LES) was done using a Smagorinski turbulence model with a constant coefficient of 0.1 . The solution is advanced forward in time with a first-order Euler time integration method. 


\section{Results}

To visualize regions of rotational flow, a sequence of iso-surfaces of vorticity in the wake of the truncated GTS is shown in Figure 4 for the coarse grid. As the solution evolves, shear layers develop on the periphery of the truncated GTS. These shear layers roll up into a vortex ring, which is advected downstream (Figure 4a). A secondary vortex ring begins to develop, though it is not quite as coherent as the first. Shortly thereafter, the vortex rings break apart and no evidence of the formation of new vortex rings is seen for the rest of the simulation. These initial transients quickly subside and the shear layers extend downstream of the base of the GTS. The shear layers undulate periodically in time and frequently shed patches of vorticity into the bluff body wake (Figure 4d). It can be seen that the massively separated wake interacts with the ground plane downstream of the GTS, resulting in a flow separation bubble on the ground plane (Figure 4d). The vorticity fields from the solution on the fine grid are slightly different in appearance than those obtained for the coarse grid. Since the turbulent length scale is dependent on the grid resolution, the vorticity fields calculated on the fine grid (Figure 5) possess significantly more small scale structures than those found for the coarse grid. Additionally, a review of the animations of the iso-vorticity surfaces reveals that patches of vorticity often burst through the shear layers in the fine grid solution, while no such phenomena is seen to occur in the coarse grid solution.

One of the more interesting calculated quantities is the helicity field in the wake of the truncated GTS. Helicity is defined to be $\mathbf{u} \times \omega$, where $\mathbf{u}$ and $\omega$ are the velocity and vorticity vector fields, respectively. The helicity can be thought of as a measure of a flow field that has a swirling nature to it. For instance, a two-dimensional viscous vortex with an axial velocity component acting co-linear with the vorticity field would be an example of a flow with a positive value of helicity. Regions of a flow field that are irrotational $(\omega=$ $0)$ or have a no-slip boundary condition $(\mathbf{u}=0)$ have a zero value of helicity. Figures 6 and 7 show iso-surfaces of the time-averaged helicity in the wake of the truncated GTS for the coarse and fine grids, respectively. The time averages are calculated over the life of the simulation after the initial transients have decayed. The surfaces denote values of helicity that are $\pm 10,000 \mathrm{~m} / \mathrm{s}^{2}$, respectively. The coherent structures present in the wake of the GTS are most clearly evident in the upstream views of the helicity iso-surfaces. It can be seen that the top corners each spawn the formation of two oppositely-signed, swirling flow structures. From the side views in Figure $6 \mathrm{~b}$ and $7 \mathrm{~b}$, it is evident that these structures are fairly robust and persist relatively far downstream in the wake of the truncated GTS. The solutions on the coarse grid and, more distinctly, on the fine grid demonstrate oppositely-signed surfaces of helicity at the bottom corners of the GTS. However, these surfaces are smaller than those being produced on the top corners, indicative of the influence of the ground plane. Due to the potential upstream influence of the truncation of the GTS geometry, the reader may be skeptical of the validity of these helicity 
surfaces and whether or not they would exist in an actual flow. To address this issue, future computational studies need to determine the sensitivity of these results to the upstream truncation condition, as well as the turbulence model and other boundary conditions used in the simulations.

\section{Comparison with Experiments}

We wanted to determine how well the simulations with the truncated geometry compare with the experimental data for the full GTS geometry [7] taken at a $R e_{w}=2.0 \times 10^{6}$ and yaw angle of $0^{\circ}$. For this comparison, the particle image velocimetry (PIV) data of the wind tunnel study [7] is employed. Figure 8 shows a schematic of the PIV setup for vertical and horizontal mid-plane velocity field measurements in the wake of the GTS. Post-processing of these velocity fields allows for the calculation of other flow quantities, such as the vorticity or kinetic energy fields.

Figure 9 shows a comparison of the time-averaged velocity fields in the vertical planes for the coarse grid and fine grid, where the time-averaged quantities are calculated in the manner described in Section 3. Figure 10 shows the corresponding PIV velocity measurements in this same plane. Due to the limited field of view of the PIV camera, only a portion of the wake of the GTS is measured in the PIV data. There is a difference in the wake structure when comparing the results of the vertical mid-plane velocity fields. For the solutions from both the coarse and fine grids, two counter-rotating flow structures exist in the separated wake, with the top one positioned slightly upstream of the bottom one. However, in the PIV data, there is no evidence for a second flow structure. Perhaps, this second flow structure exists outside the field of view of the PIV camera. The measured wake in the PIV data looks very similar to the near wake of a bus [9]. This distinct difference between the simulations and experimental measurements may be due to the effects of truncating the model or neglecting the undercarriage on the GTS geometry (e.g., the four supporting posts on the GTS model) and the wind tunnel walls.

Figure 11 shows instantaneous fields of the y-component of vorticity in the horizontal mid-plane for the coarse grid, fine grid, and PIV data. As expected, there is a distinct difference between the vorticity fields of the coarse and fine grids, namely that the fine grid captures more smaller scale structures in the shear layers being shed by the GTS. These small scale structures are also evident in the shear layers measured in the PIV data.

\section{Conclusions}

Unsteady, three-dimensional, large-eddy simulations are done on the flow about a truncated GTS geometry located above a no-slip surface. Calculations of the vorticity field show the unsteady behavior of the shear layers being shed 
by the GTS geometry. Time-averaged iso-surfaces of helicity demonstrate the existence of coherent flow structures that are formed from the corners of the GTS. These structures do not readily dissipate and exist beyond the massively separated wake. A comparison with the PIV velocity measurements made at the same Reynolds number and yaw angle reveals differences in the structure of the separated wake in the vertical mid-plane, perhaps indicating the influence of not including the model supports or of truncating the GTS model. Future simulations on the GTS geometry must investigate the sensitivity of the solutions to these effects, including the influence of the wind tunnel confinement, turbulent boundary layer treatment, and time averaging issues. In regard to achieving the goal of reducing the drag of heavy vehicles, future simulations also need to explore and to understand the flow physics of various base-drag reduction concepts.

\section{References}

1. Lanser, W.R., Ross, J.C., \& Kaufman, A.E. Aerodynamic Performance of a Drag Reduction Device on a Full-Scale Tractor Trailer. Paper No. 912125, SAE Transactions, 1991.

2. Virgin, B. Rounded add-on makes driving truck less of a drag, Seattle PostIntelligencer, http://seattlepi.nwsource.com/business/103544_truck10. shtml, January 10, 2003.

3. Whitten, W.D. Collapsible Streamlined Tail for Trucks and Trailer, U.S. Patent Number 6,309,010, September 27, 2000.

4. Bassett, D.A., Bassett, M.R., \& Schroeder, J.C. Method and Apparatus for Reducing Drag on a Moving Body, U.S. Patent Number 5,374,013, November 4, 1993.

5. Kirsch, J.W., Sabodh, K.G., \& Garg, K. Airvane Device for Bluff Vehicles and the Like, U.S. Patent Number 3,999,797, November 26, 1975.

6. Engler, R.J. Advanced Aerodynamic Devices to Improve the Performance, Economics, Handling and Safety of Heavy Vehicles, SAE Paper 2001-01-2072.

7. Storms, B.L., Ross, J.C., Heineck J.T., Walker, S.M., Driver, D.M. \& Zilliac, G.G. An Experimental Study of the Ground Transportation (GTS) Model in the NASA Ames 7- by 10-ft Wind Tunnel, NASA/TM-2001-209621, February, 2001.

8. Gresho, P.M. \& Sani, R.L Incompressible Flow and the Finite Element Method, Volumes 1 \& 2, April, 2000.

9. Gotz, H. \& Mayr, G. Commercial Vehicles, Aerodynamics of Road Vehicles, edited by Hucho, W.H., 1998, 415-488. 


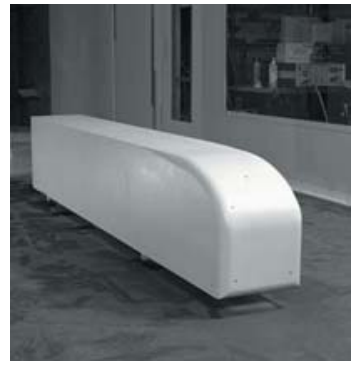

(a)

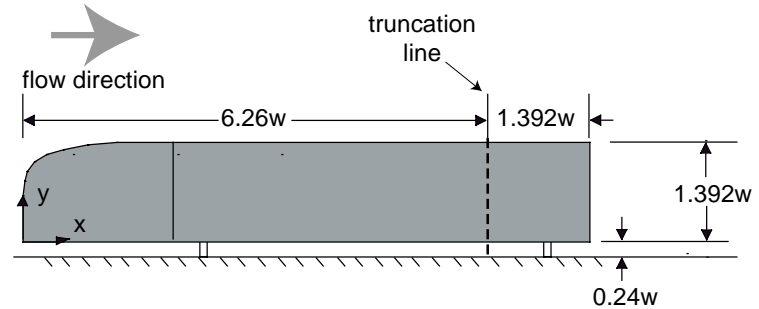

(b)

Fig. 1. (a) Photo of the GTS geometry in the NASA Ames $7 \mathrm{ft} \times 10 \mathrm{ft}$ wind tunnel. (b) Schematic of the GTS geometry and location of the model truncation. $w$ is the width of the GTS model $(0.3238 \mathrm{~m})$.

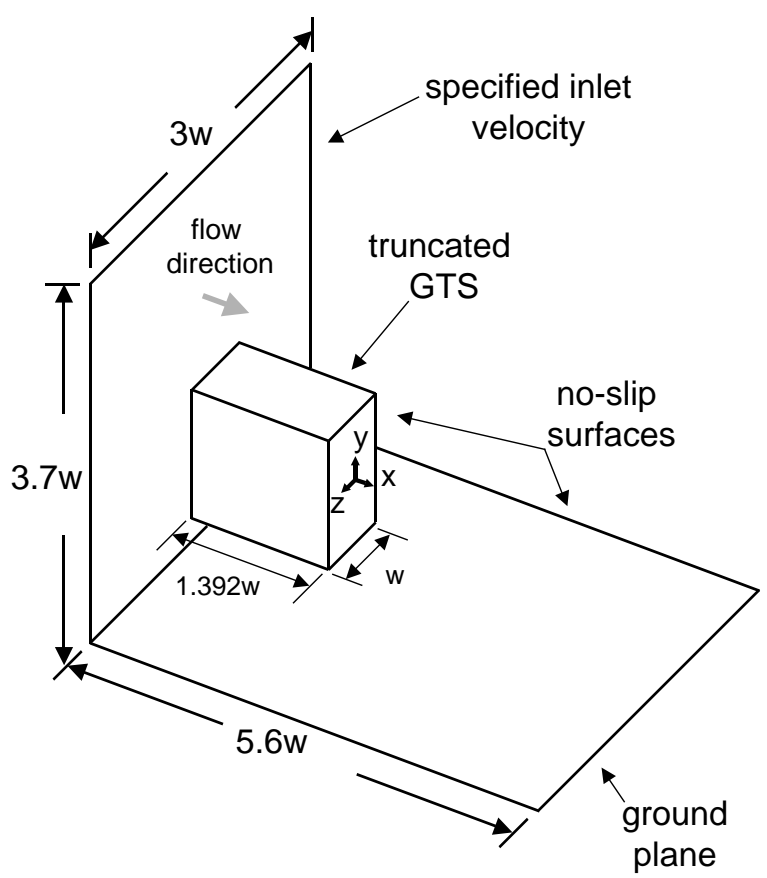

Fig. 2. Computational domain and boundary conditions for the flow simulations. 

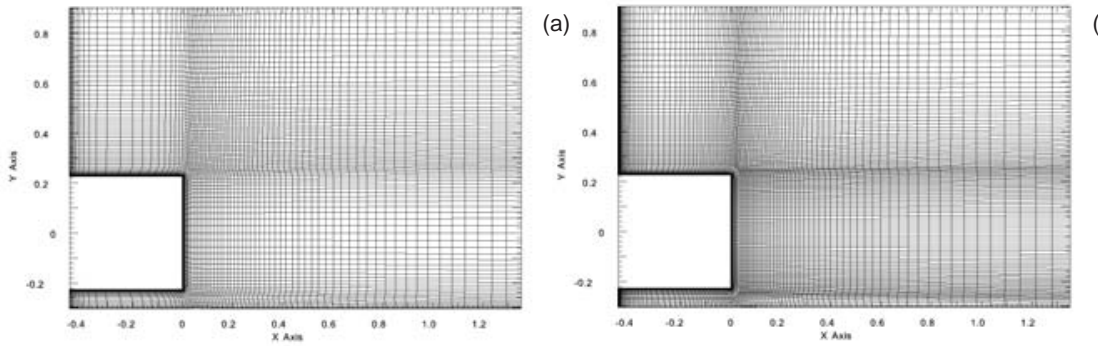

Fig. 3. Vertical mid-plane cross-sections $(z=0)$ of the $(a)$ coarse and (b) fine grids used in the computational study.
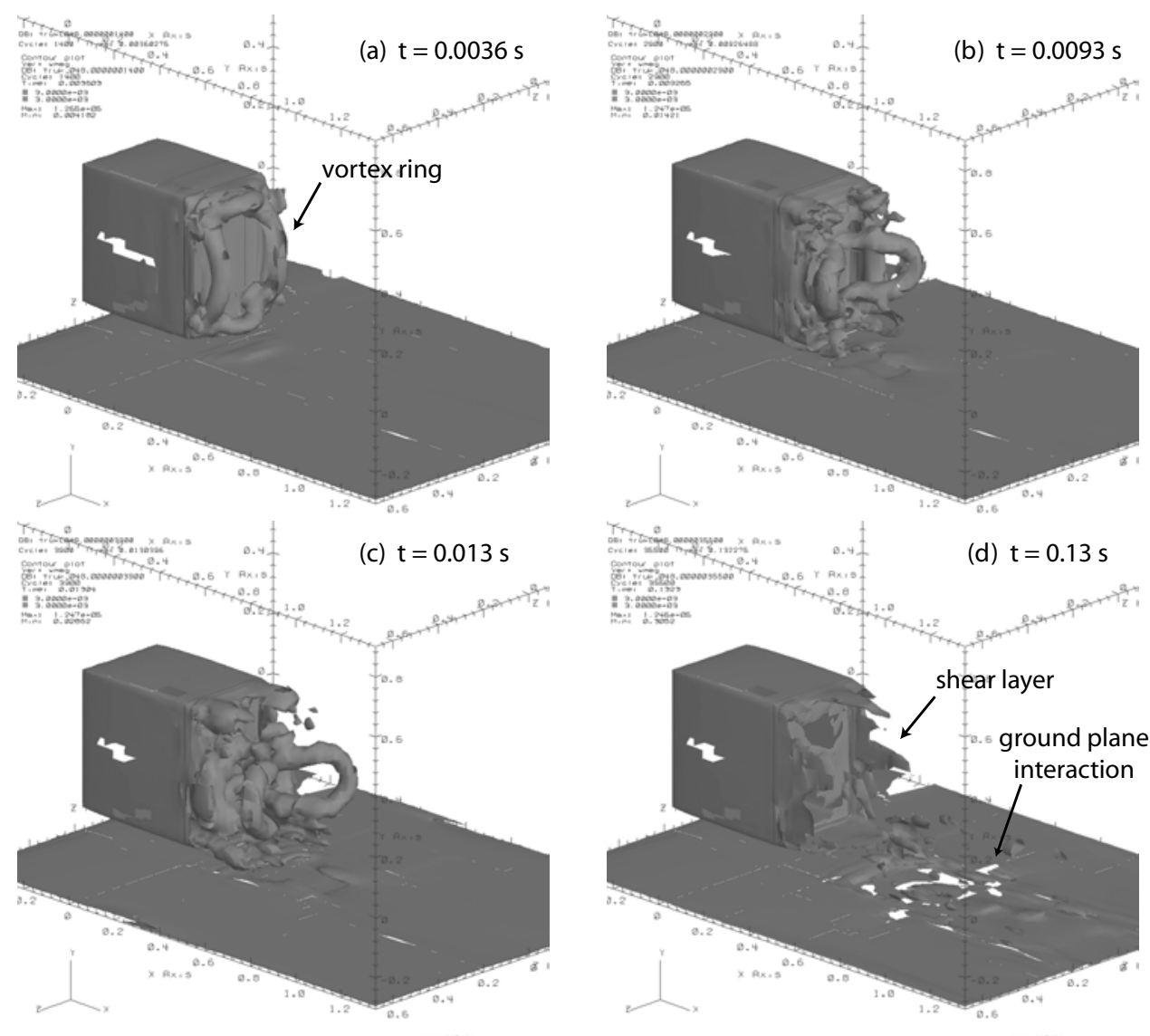

Fig. 4. Iso-surfaces of the vorticity magnitude at several times in the coarse grid simulation. 

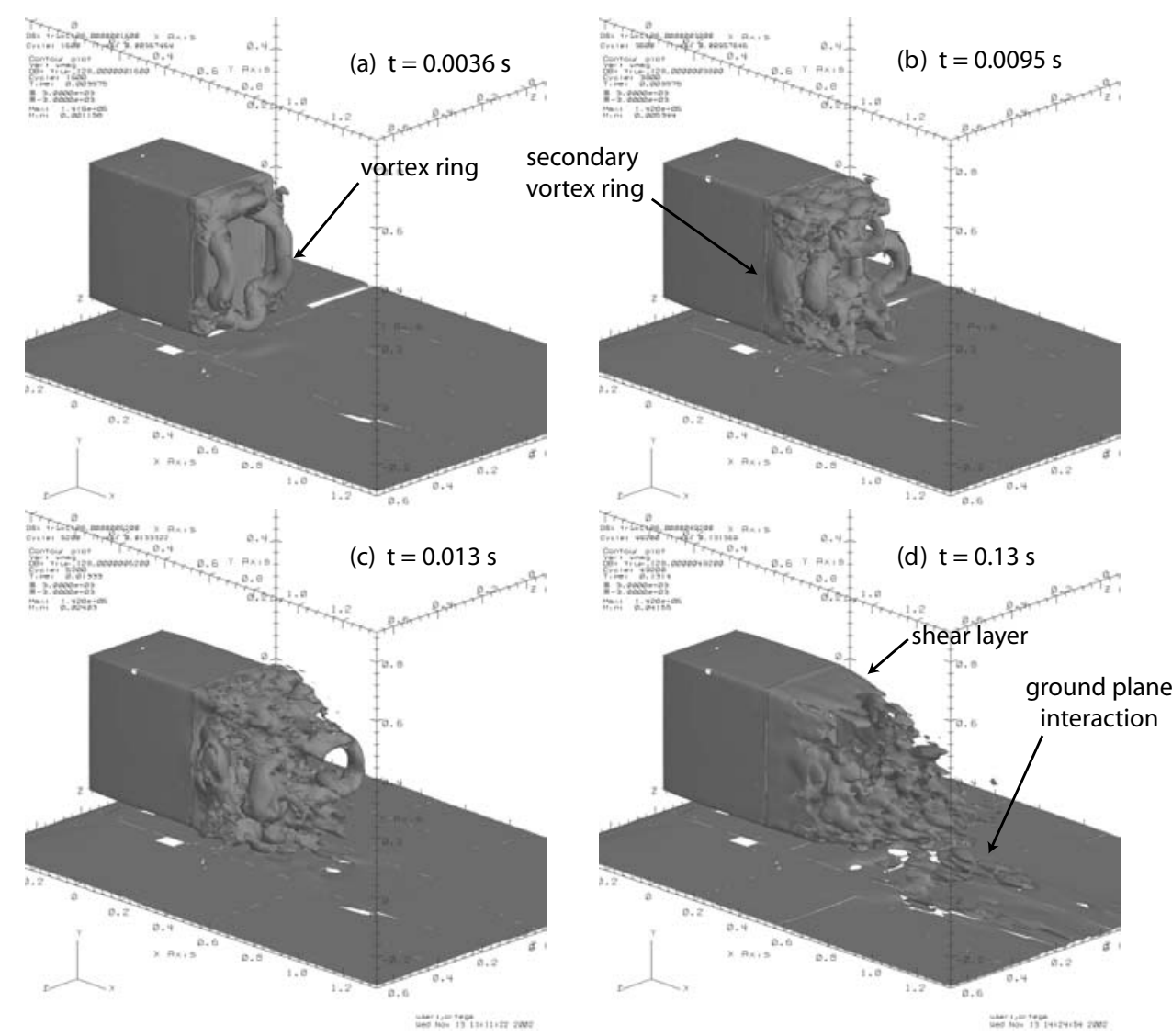

Fig. 5. Iso-surfaces of the vorticity magnitude at several times in the fine grid simulation. 


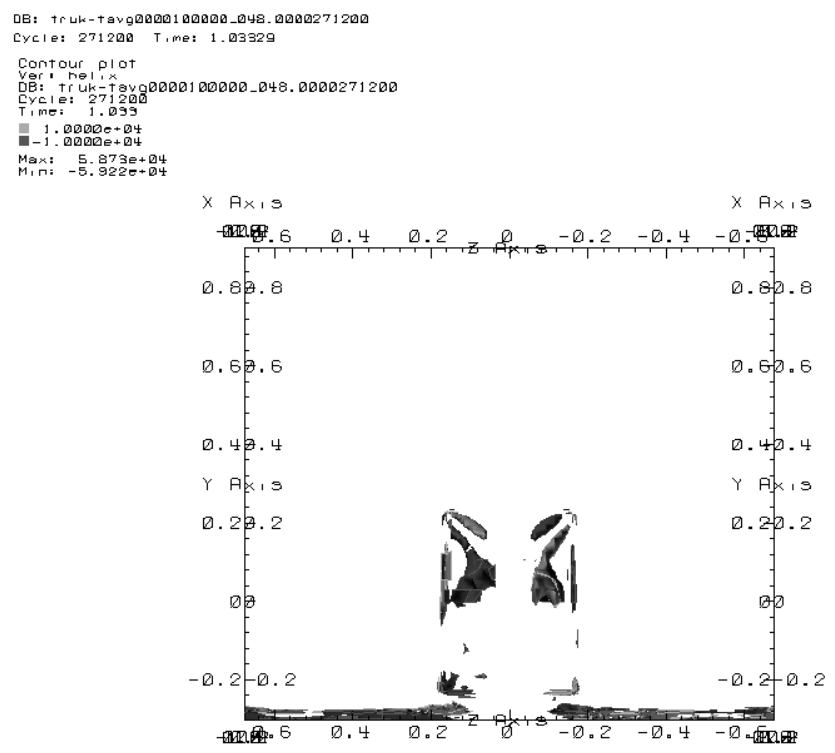

(a)

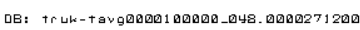

Cycle: 271200 T.me: 1.03329

Contour plot

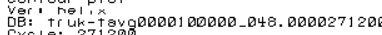

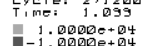

Max: $-5.873 e+04$

(b)

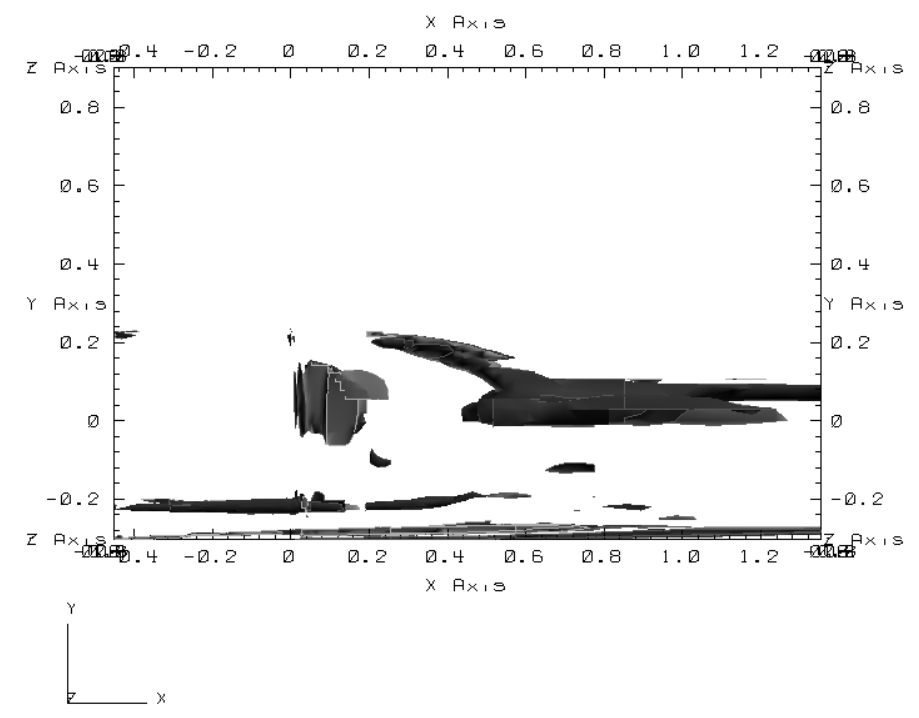

User : vor tega
Wed Nav E 13:48:05 2002

Fig. 6. (a) Upstream and (b) side views of the time-averaged iso-surfaces of helicity for the coarse grid. 


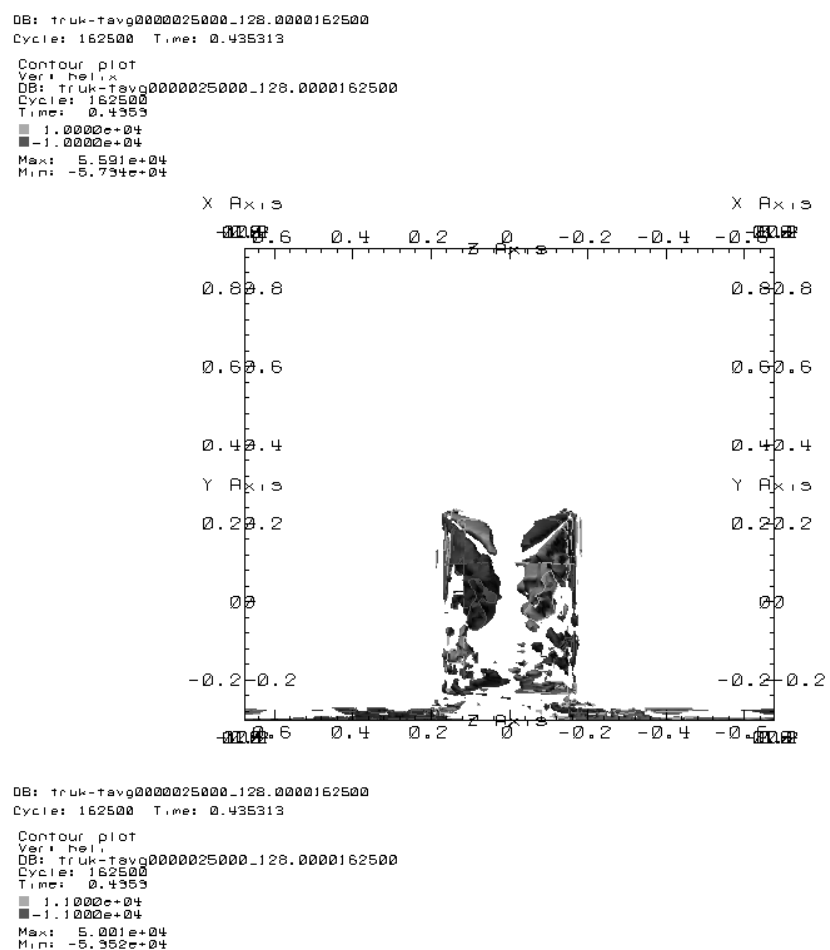

(a)

Contour plo

Tycie: 1625

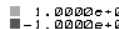

Min:

(b)

Max: 5 . $9019+10$

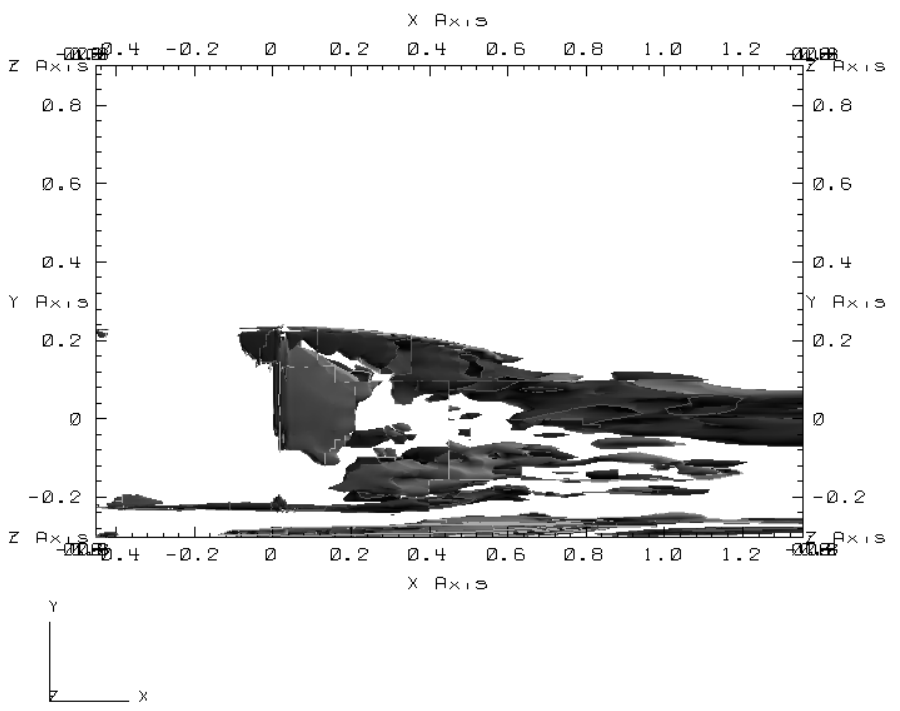

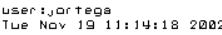

Fig. 7. (a) Upstream and (b) side views of the time-averaged iso-surfaces of helicity for the fine grid. 


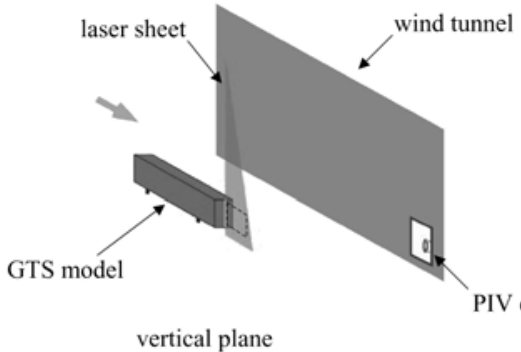

(a)

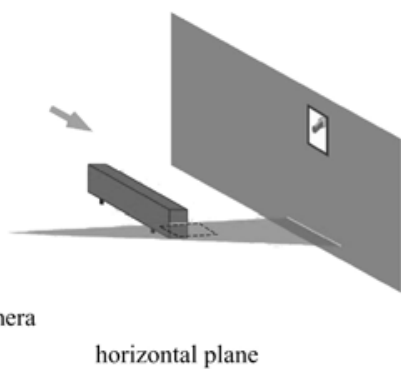

(b)

Fig. 8. Schematic of the PIV setup in the NASA Ames $7 \mathrm{ft} \times 10 \mathrm{ft}$ wind tunnel for (a) vertical mid-plane and (b) horizontal mid-plane measurements.

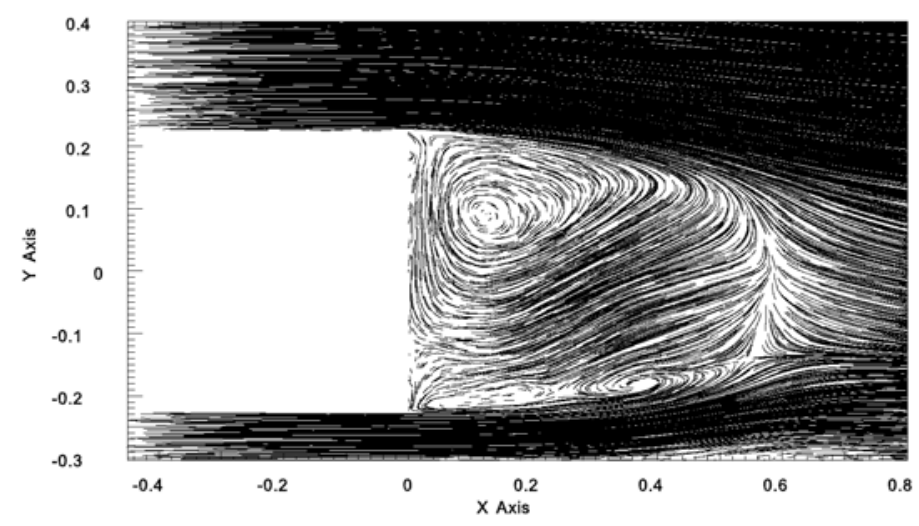

(a)

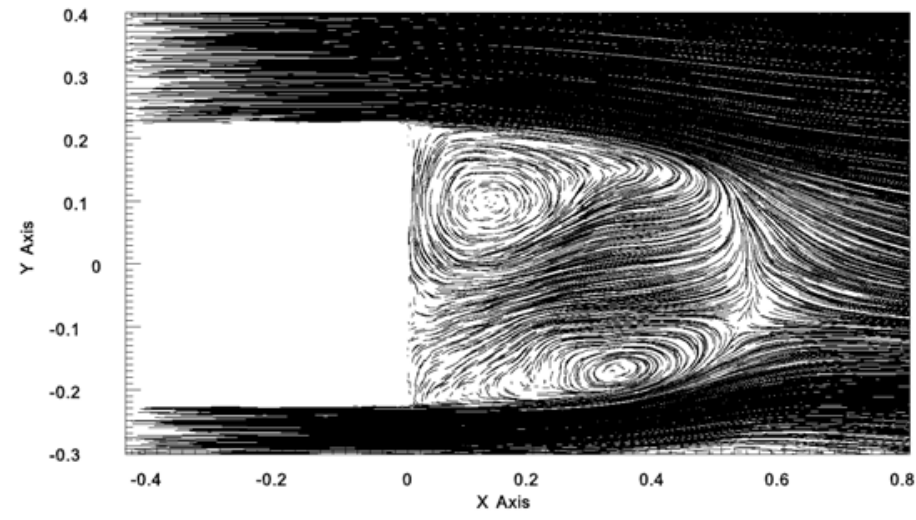

(b)

Fig. 9. Time-averaged velocity fields in the vertical mid-plane for the (a) coarse and (b) fine grids. 

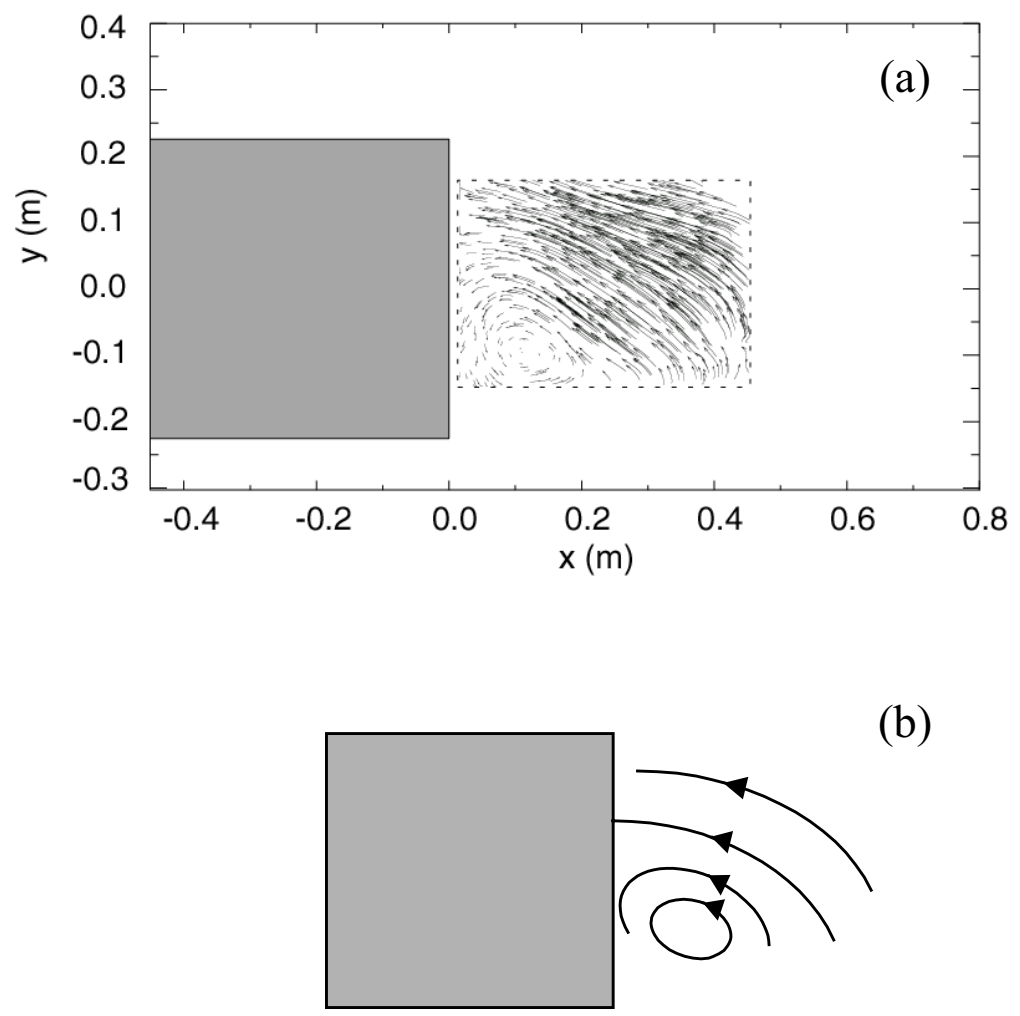

(b)

Fig. 10. (a) PIV velocity field measurements in the vertical mid-plane. For clarity, a schematic of the velocity field in (a) is shown in (b). 


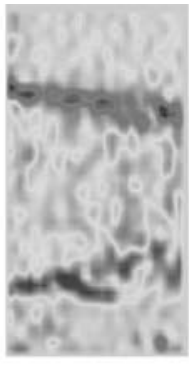

(a)

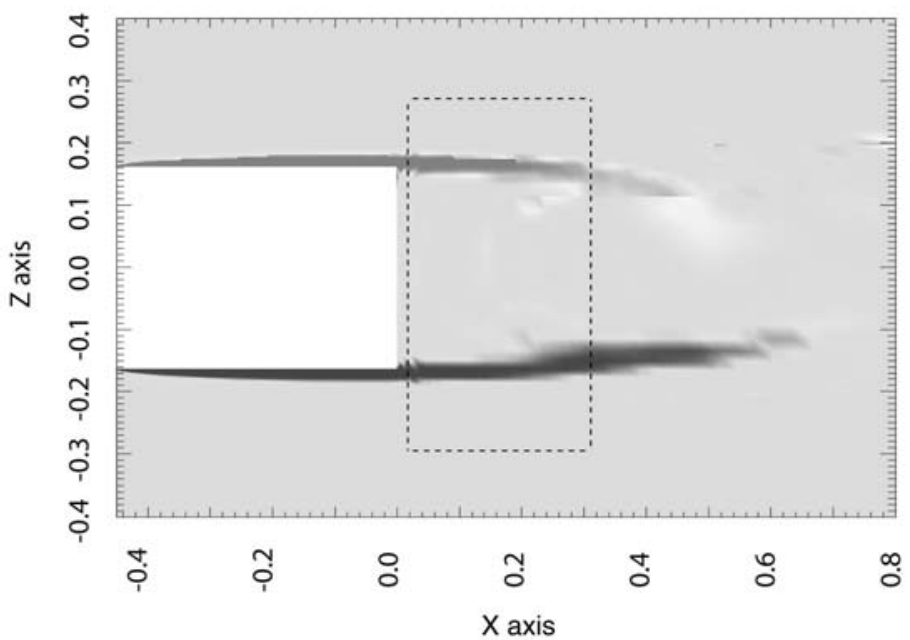

(b)

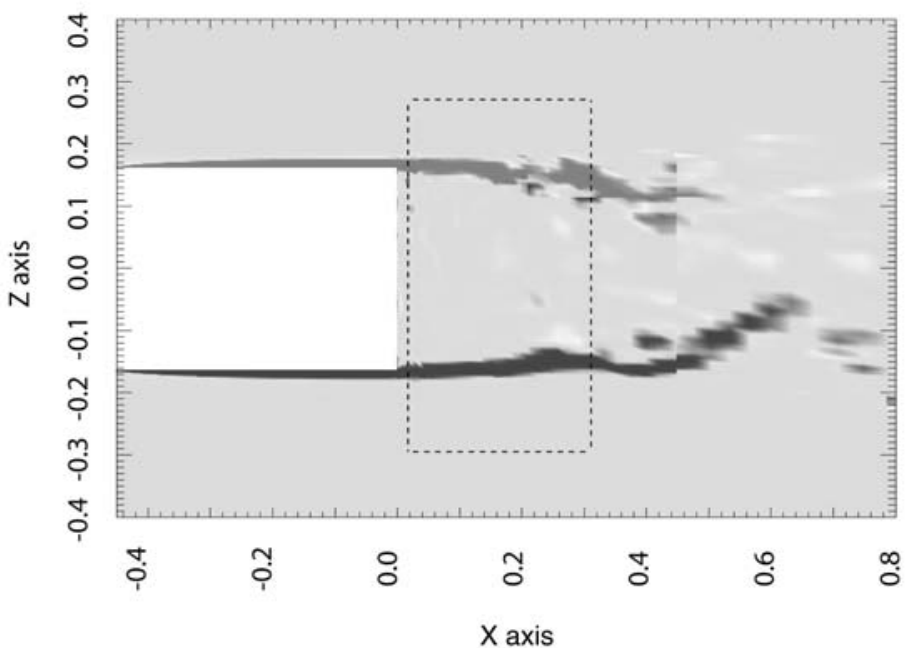

(c)

Fig. 11. Instantaneous y-component of vorticity in the horizontal mid-plane for the (a) PIV measurements, (b) coarse grid, and (c) fine grid. The dashed regions in (b) and (c) correspond to the region shown in (a). 